Journal de la Société des américanistes

Journal de la Société

des Américanistes

$87 \mid 2001$

tome 87

\title{
Henry Reichlen (1914-2000)
}

\section{Marie-France Fauvet-Berthelot}

\section{OpenEdition}

Journals

Édition électronique

URL : http://journals.openedition.org/jsa/2218

DOI : $10.4000 /$ jsa. 2218

ISSN : 1957-7842

\section{Éditeur}

Société des américanistes

\section{Édition imprimée}

Date de publication : 1 janvier 2001

Pagination : 396-400

ISSN : 0037-9174

\section{Référence électronique}

Marie-France Fauvet-Berthelot, « Henry Reichlen (1914-2000)», Journal de la Société des américanistes [En ligne], 87 | 2001, mis en ligne le 17 novembre 2005, consulté le 15 septembre 2020. URL : http:// journals.openedition.org/jsa/2218 
Henry REICHLEN (1914-2000)

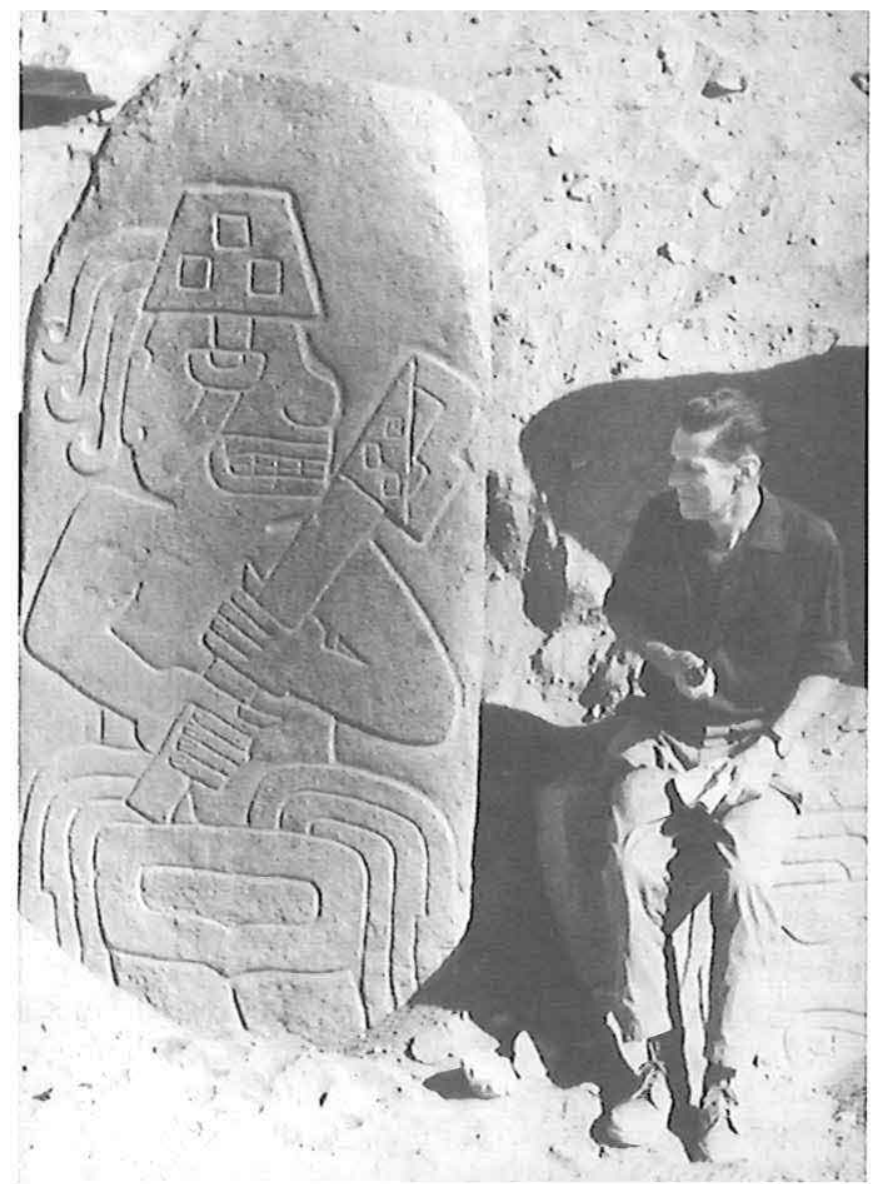

Henry Reichlen à Cerro Sechin (Pérou) en 1965

Henry Reichlen s'est éteint à Paris le 8 décembre 2000. De nationalité suisse, il est né le 10 avril 1914 à Bulle (canton de Fribourg). Après des études de droit, d'histoire de l'art, de botanique, de géologie et de zoologie à l'université de Fribourg, il est, de 1934 à 1937, attaché au musée cantonal d'Histoire naturelle et d'Archéologie de Fribourg où il étudie et complète les collections de géologie, botanique et entomologie des 
Pré-alpes fribourgeoises, en même temps qu'il réalise des fouilles sur des sites lacustres néolithiques du canton de Fribourg. Sa carrière ensuite se partagera entre l'Amérique du Sud et le musée de l'Homme à Paris.

En 1938, chargé de mission par le musée d'Histoire naturelle de Fribourg, il part pour l'Argentine où il rencontre les frères Emilio et Duncan Wagner qui avaient effectué, en 1927, des fouilles dans la région de Santiago del Estero. Ces derniers l'encouragent à reprendre des fouilles, ce qu'il fera d'octobre 1937 à août 1938 à Averias et Icano ; il est alors assistant technique au musée archéologique de Santiago del Estero. Puis il rentre en Europe en 1939, où il suit des cours d'ethnologie à l'université de Fribourg et à Paris à l'Institut d'ethnologie, à l'École pratique des hautes études et à l'Institut de paléontologie humaine. À cette date, il devient membre de la Société des Américanistes; il sera membre de son conseil de 1943 à 1977.

En 1940, il est nommé par Paul Rivet responsable par intérim du département d'Amérique du musée de l'Homme, charge qu'il conserve jusqu'en 1947. Dans ce musée, il s'occupe de la réinstallation des salles d'exposition d'Amérique, d'Océanie et des peuples arctiques et réorganise avec grand soin les collections archéologiques du Pérou conservées dans ce département .C'est à cette époque qu'il épouse Paule Barret, anthropologue physique, collaboratrice de Paul Rivet pendant de longues années, avec laquelle il publiera souvent en collaboration. En 1945, il intègre le CNRS nouvellement créé et sera nommé maître de recherches en 1963.

Il retourne ensuite en Amérique du Sud. De 1947 à 1952, il fait partie de la mission ethnologique française au Pérou septentrional organisée par Paul Rivet. Dans ce cadre, il effectue une série de reconnaissances et de fouilles archéologiques dans le sud-est du département de Cajamarca, région peu connue sur le plan archéologique. Il travaille sur les sites de Wayrapong, où il trouve une intéressante industrie lithique, Las Torrecitas, mettant au jour une céramique chavinoïde, ainsi qu'à La Vaqueria et Chondorko, chantiers qui lui permettent d'établir la séquence céramique de Cajamarca qui fait toujours date. Il fait ensuite une mission dans le nord du Pérou, dans la vallée du Haut-Utcubamba (département de Amazonas) et réalise des fouilles dans les cités semi-fortifiées d'altitude de Kuelape et San Pedro de Washpa ; à Chipurik et Ayshpachaca, il découvre des monuments funéraires anthropomorphes et à Revash s'intéresse aux maisons funéraires collectives. Il complète ce travail archéologique par des enquêtes linguistiques et ethnographiques. Dans le même temps, il recueille une importante documentation photographique sur les gravures et peintures rupestres du versant occidental des Andes, notamment celles de Toro Muerto (département d'Arequipa) dans le sud, classées aujourd'hui monument national. À la fin de l'année 1958, il rejoint en Patagonie chilienne Annette Emperaire dont le mari, l'ethnologue José Emperaire, vient de décéder accidentellement sur le site de Ponsonby. Il participe aux fouilles de ce site et à celles de la grotte Fell et de la grotte Oosin Aike, aidant ainsi Annette Emperaire à clore cette campagne de fouilles.

Les centres d'intérêt d'Henry Reichlen étaient multiples - il a allié, on l'a vu, dès sa formation universitaire, sciences naturelles et sciences humaines. Outre des articles concernant ses travaux de terrain archéologiques et ethnographiques en Argentine et au Pérou, il a publié quelques articles sur des collections du musée de l'Homme provenant de la Martinique, de la Californie, du Pérou, de l'Amazonie. Il portait également un grand intérêt à l'histoire des collections et a consulté de nombreuses 
archives à Paris, notamment sur les anciennes missions françaises. On lui doit la publication, dans le Journal des Américanistes, du manuscrit Boscana conservé à la Bibliothèque nationale de Paris, manuscrit considéré par $\mathrm{A}$. Kroeber comme le document ethnographique le plus important laissé sur les Indiens de Californie par les franciscains qui les convertirent de 1812 à 1822 .

Homme de musée et homme de terrain, affable, courtois, méticuleux, passionné par les objets, il a considérablement enrichi le patrimoine français par le don de collections archéologiques et ethnographiques au département Amérique du laboratoire d'ethnologie. Il a également fait don de quelques objets aux départements d'Océanie, d'Afrique du Nord et d'Europe, souvent acquis dans des ventes aux enchères, qu'il fréquentait beaucoup.

Son autre passion était la peinture et la gravure contemporaines : lui-même, issu d'une famille de peintres, était un bon dessinateur.

Sa fille Marie-Hélène a fait don de ses papiers et de ses photographies au musée de l'Homme.

\section{Marie-France FAUVET-BERTHELOT}

Musée de l'Homme

\section{PRINCIPALES PUBLICATIONS D'HENRY REICHLEN}

1940. - « Recherches archéologiques dans la province de Santiago del Estero, Argentine », Journal de la Société des Américanistes, XXXII, pp. 133-225.

1940. - "Contribution à l'archéologie de la Martinique. Le gisement de l'Anse-Belleville », Journal de la Société des Américanistes, XXXII, pp. 227-274 (en collaboration avec Paule Barret).

1941. - « Étude technologique de quelques objets d'or de Lambayeque, Pérou », Journal de la Société des Américanistes, XXXIII, pp. 119-154.

1941. - « Contribution à l'archéologie de la Martinique. Le gisement de Paquemar », Journal de la Société des Américanistes, XXXIII, pp. 91-117 (en collaboration avec Paule Barret).

1942. - « Contribution à l'étude de la métallurgie précolombienne de la province d'Esmeraldas, Équateur », Journal de la Société des Américanistes, XXXIV, pp. 201-228.

1943-1946. — "Contribution à l'archéologie de la Guyane française », Journal de la Société des Américanistes, XXXV, pp. 1-24 (en collaboration avec Paule Barret).

1947. - « Note sur l'origine d'une sculpture mexicaine du musée de l'Homme », Journal de la Société des Américanistes, XXXV, pp. 177-180.

1947. - Les momies, Paris, Éditions Prisma (en collaboration avec le Dr Dérobert).

1949. - « Recherches archéologiques dans les Andes de Cajamarca. Premier rapport de la Mission ethnologique française au Pérou septentrional ", Journal de la Société des Américanistes, XXXVIII, pp. 137-174 (en collaboration avec Paule Reichlen).

1950. - « Deux chiens pré-hispaniques du Désert d'Atacama. Recherches anatomiques sur le chien des Incas ", Travaux de l'Institut français d'études andines, Lima, II, pp. 1-18 (en collaboration avec M. Friant).

1950. — « Étude de deux fardeaux funéraires de la côte centrale du Pérou », Travaux de l'Institut français d'études andines, Lima, II, pp. 39-50. 
1950. — " Mutilations dentaires sur des crânes indiens de Tocarji, Bolivie ", Journal de la Société des Américanistes, XXXIX, pp. 183-186.

1950. - « Recherches archéologiques dans les Andes du haut Utcubamba. Deuxième rapport de la Mission ethnologique française au Pérou septentrional », Journal de la Société des Américanistes, XXXIX, pp. 219-246 (en collaboration avec Paule Reichlen).

1950. — «Illustration d'un rite précolombien de divination (un wako de Trujillo) », Journal de la Société des Américanistes, XXXIX, pp. 258-259.

1953. - «Fêtes, danses et rites des Indiens de Cajamarca », Journal de la Société des Américanistes, XLII, pp. 391-413.

1954. — « Les collections américaines du musée d'Angers », Journal de la Société des Américanistes, XLIII, pp. 161-171.

1954. - Le Pérou et la Bolivie, Paris, Éditions d'Art Lucien Mazenod, coll. Les sculpteurs célèbres, pp. 122-125.

1956. - Incas, Mayas et Aztèques, Paris, Édition des Deux-Mondes, pp. 3-24.

1957. — «Précis d'histoire de l'art. Les civilisations précolombiennes », in : Encyclopédie Larousse, l'Art et l'Homme, René Huyghe (ed.), pp. 107-114.

1958. - « La chunga de Cajamarca. Contribution à l'étude des jeux », Miscellanea Paul Rivet Octogenario decata, Mexico, pp. 487-498 (en collaboration avec Paule Reichlen).

1961. - « Vues nouvelles sur les civilisations précolombiennes du Pérou », Cahiers de l'Institut des hautes études de l'Amérique latine, $\mathrm{n}^{\circ} 2$, pp. 39-56.

1961. - «Objets en bois sculpté du Bas Amazone ", Objets et Mondes, Paris, I, fasc. 2, pp. 17-30.

1961. - « À propos des découvertes d'urnes en pierre dans les Andes de Pomabamba (Ancash) », Travaux de l'Institut français d'études andines, Paris-Lima,VIII, pp. 111-118.

1961. — « Les " tejos ” à Moche et à Cajamarca. Contribution à l'étude des jeux au Pérou ", Travaux de l'Institut français d'études andines, Paris-Lima, VIII, pp. 53-58 (en collaboration avec Paule Reichlen).

1962. — « Un bijou d'or de Lambayeque, Pérou », Objets et Mondes, Paris, II, fasc. 2, pp. 77-84.

1963. - « La Grotte Fell et autres sites de la région volcanique de la Patagonie chilienne », Journal de la Société des Américanistes, LII, pp. 167-254 (en collaboration avec José Emperaire et Annette Laming-Emperaire).

1963. — « La mission de Léon Cessac en Californie (1877-1879) », Objets et Mondes, Paris, III, fasc., pp. 17-34 (en collaboration avec R. Heizer).

1964. - «The scientific expedition of Leon de Cessac to California, 1877-1879 », Berkeley, Reports of the University of California, Archaeological survey, $\mathrm{n}^{\circ}$ 61, pp. 9-23 (en collaboration avec Robert F. Heizer).

1965. - « Dos telas pintadas del Norte del Perú », Revista peruana de Cultura, Lima, pp. 6-16. 1965. - « L'or dans le Nouveau Monde » in : Splendeurs de l'or , P. Amiet \& F. Balsan (ed.), Paris, Hachette, pp. 62-75.

1967. — « Reconocimiento arqueológico en los Andes de Cajamarca », Cuadernillos de antropología, I, Universidad nacional mayor de San Marcos, Lima, 28 p.

1971. - « Le manuscrit Boscana de la Bibliothèque Nationale de Paris. Relation sur les Indiens Acâgchemem de la Mission de San Juan Capistrano, Californie ", Journal de la Société des Américanistes, XL, pp. 233-273 (en collaboration avec Paule Reichlen). 
1975. - Le monde étrange des momies, Paris, éditions Pygmalion, 160 p. (en collaboration avec le Dr Dérobert).

1975. - « Note sur l'archéologie du Pérou », in : Les Guides Bleus, Pérou, Paris, Hachette, pp. $35-59$. 